\title{
LUXAÇÃO PATELAR MEDIAL GRAU IV EM GATO: RELATO DE CASO
}

\author{
ALVES, Endrigo Gabellini Leonel ${ }^{1}$ \\ FARIA, Rogério Rodrigues Arantes ${ }^{2}$ \\ VARON, Jéssica Alejandra Castro ${ }^{3}$ \\ ROSADO, Isabel Rodrigues ${ }^{1}$ \\ REZENDE, Cleuza Maria de Faria ${ }^{4}$
}

Recebido em: 2015.07 .23

Aprovado em: 2017.10.05

ISSUE DOI: $10.3738 / 21751463.1542$

\begin{abstract}
RESUMO: Este trabalho tem como objetivo relatar um caso de luxação patelar medial grau IV em gato, abordando aspectos clínicos e cirúrgico. A luxação de patela caracteriza-se por sua saída do sulco troclear. Comumente essa patologia é congênita e se não tratada precocemente pode gerar graves alterações osteomusculares. A luxação patelar não é comum em gatos especialmente o grau IV. Foi atendido um gato com histórico de claudicação, apoio parcial e atrofia do membro pélvico esquerdo. No exame físico foi observada a patela luxada medialmente de forma irredutível, incapacidade de extensão completa do membro e desvio medial da tuberosidade tibial. O exame radiográfico mostrou a patela luxada medialmente e desvios angulares de fêmur distal e tíbia proximal. No tratamento cirúrgico foram realizados trocleoplastia, desmotomia medial da cápsula articular, liberação parcial do reto femoral e vasto medial, transposição da tuberosidade tibial, sutura antirotacional, imbricação lateral de cápsula articular e reforço do retináculo lateral com fascia lata. No pós-operatório o animal permaneceu com bandagem de Robert Jones por dez dias e recebeu como medicação meloxicam, cefalexina e condroton. O tratamento foi eficiente no realinhando do mecanismo extensor e na manutenção da patela no sulco troclear. O animal voltou a utilizar o membro aos 10 dias de pós-operatório. Conclui-se que a associação de técnicas cirúrgicas realizadas promove o alinhamento das estruturas do mecanismo extensor e a estabilidade da patela no sulco troclear, sendo eficiente no tratamento da luxação patelar medial em gatos.
\end{abstract}

Palavras-chave: ortopedia, Felis cantus, técnica cirúrgica.

SUMMARY: This study aimed to report a grade IV medial patellar luxation in cat, addressing clinical and surgical aspects. Its dislocation from the trochlear groove characterizes the patellar luxation. Commonly this condition is congenital and if not treated early can cause serious musculoskeletal disorder. The patellar luxation is not common in cats especially the degree IV. A cat with a history of claudication, partial support weight and atrophy of the left hindlimb was granted. The physical examination was observed irreducible patellar dislocation, incomplete extension member and medial deviation of the tibial tuberosity. Radiographic examination showed the patella dislocated medially and angular deviation of the distal femur and proximal tibia. In the surgical treatment were performed, trochleoplasty, medial capsular release, partial release of the rectus femoris and vastus medialis mucles, tibial tuberosity transposition, anti-rotational suture, lateral capsular imbrication and strengthening the lateral retinaculum with fascia lata. Postoperatively the animal remained with Robert Jones bandage for ten days and received meloxicam, cephalexin and condroton as medication. The treatment was effective in realigning the extensor mechanism and maintenance of the patella in the trochlear groove. The animal returned to use the limb at 10 days postoperatively. It is concluded that the combination of surgical techniques performed promotes alignment of the extensor mechanism and the stability of the patella in the trochlear groove, being effective in the treatment of the medial patellar luxation in cats.

Keywords: orthopedics, Felis cantus, surgical technique.

\footnotetext{
${ }^{1}$ Professor da UNIUBE

${ }^{2}$ Graduando da Universidade de Uberaba

${ }^{3}$ Doutoranda da UFMG

${ }^{4}$ Professora da UFMG especialista em ortopedia veterinária
} 


\section{INTRODUÇÃO}

A luxação patelar é caracterizada pela saída da patela do sulco troclear para o lado medial ou lateral de forma permanente ou recorrente (DOKIC et al., 2015). É uma patologia muito comum em cães de pequeno porte afetando principalmente em animais das raças Poodle, Pinscher e Yorkshire (LARA et al., 2013) mas também pode ocorrer em cães de médio e grande porte (SOUZA et a., 2010) e em gatos (HOULTON;MEYNINK, 1989). Em gatos acredita-se que as raças Rex e Abissínio são mais acometidas (SMITH, 2004). O deslocamento medial da patela ocorre com maior frequência correspondendo a cerca de 75 a $80 \%$ dos casos de luxação patelar em cães com acometimento de bilateral em 20 a 25\% dos casos. Poucos são os estudos em gatos, mas uma distribuição semelhante a dos cães tem sido observada com maior ocorrência da luxação medial bilateral (PIERMATTEI et al., 2009). A luxação patelar medial pode ser congênita com caráter hereditário ou adquirida de forma traumática sendo a primeira mais comum representando cerca de $98 \%$ dos casos em gatos. A luxação patelar pode apresentar quatro níveis de graduação sendo classificada de acordo com as alterações presente na articulação fêmuro-tíbio-patelar (SMITH, 2004). Frequentemente a luxação patelar medial está associada a outras anormalidades musculoesqueléticas como coxa vara, redução do ângulo de antiversão do colo femoral, deslocamento medial do quadríceps femoral, rotação lateral do fêmur e medial da tíbia (SCHULZ, 2014). Todas essas alterações podem levar a distribuição anormal de força nos ossos no membro pélvico o que pode desencadear a crescimento ósseo anormal em animais jovens levando a graves alterações anatômicas. O tratamento da luxação patelar é cirúrgico e visa manutenção da patela no sulco troclear e o alinhamento do mecanismo extensor (PIERMATTEI et al., 2009).

Devido à gravidade da enfermidade e a escassez de estudos sobre a luxação patelar em felinos, este trabalho tem como objetivo relatar um caso de luxação patelar medial grau IV em gato abordando aspectos clínicos e cirúrgicos importantes para o diagnóstico e tratamento da doença.

\section{RELATO DE CASO}

Foi atendido no Hospital Veterinário da UFMG, um gato, sem raça definida, com doze meses de idade e massa corporal de $4,75 \mathrm{Kg}$. O proprietário adotou o animal adulto com dificuldade de locomoção. Na inspeção do animal em repouso e em movimento observou-se rotação medial do membro pélvico esquerdo (Fig.1.A) com apoio em semiflexão e incapacidade de extensão completa. No exame físico, notou-se limitação na extensão do membro, discreta 
atrofia muscular e patela deslocada medialmente (Fig.1.A) sem possibilidade de redução manual. A tuberosidade tibial encontrava-se desviada medialmente com uma angulação de cerca de $75^{\circ}$ (Fig.1.A). Foi então dado o diagnóstico de luxação medial de patela grau quatro. $\mathrm{O}$ exame radiográfico foi realizado e mostrou deslocamento medial da patela, discreta curvatura medial da extremidade distal do fêmur e discreta curvatura lateral da extremidade proximal da tíbia, mas não foram observadas alterações radiográficas que sugerissem doença articular degenerativa (Fig.1.B).

Figura 1: Foto dos joelhos de um gato com luxação patelar medial grau IV

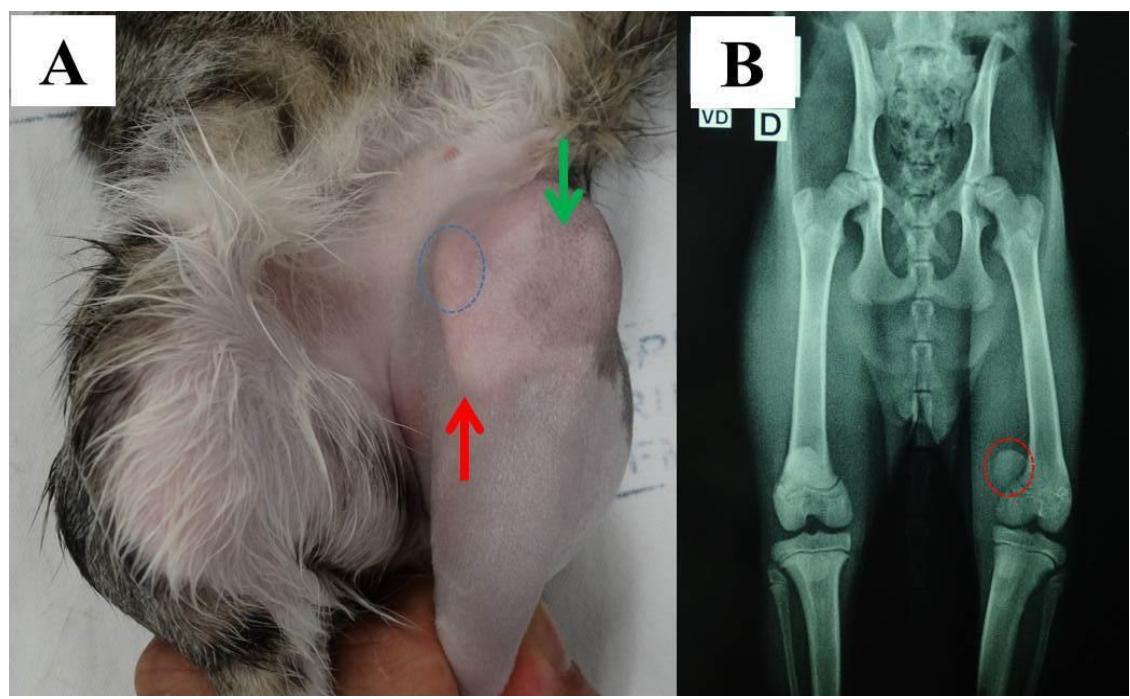

Notar na imagem "A" a patela esquerda deslocada medialmente (círculo azul), tuberosidade tibial desviada medialmente a cerca de $75^{\circ}$ (seta vermelha) e o sulco troclear (seta verde). (B) Imagem radiográfica ventro-dorsal dos membros pélvicos no mesmo animal. Observar a patela deslocada medialmente (círculo vermelho), a extremidade distal do fêmur com discreta curvatura medial e a extremidade proximal da tíbia com discreto desvia lateral.

O animal foi encaminhado para cirurgia para redução da luxação patelar e realinhamento do mecanismo extensor. Realizou-se a preparação do membro para uma cirurgia asséptica procedendo-se ampla tricotomia e higienização do local cirúrgico. Em seguida o animal foi encaminhado para o bloco cirúrgico onde recebeu cetamina $(10 \mathrm{mg} / \mathrm{kg}$, IM), midazolam $(0,5$ $\mathrm{mg} / \mathrm{kg}$ IM) e metadona $(0,5 \mathrm{mg} / \mathrm{kg} \mathrm{IM})$ como medicação pré-anestésica. A indução foi realizada com propofol (5mg/kg IV) e manutenção isofurano diluído em $100 \%$ de oxigênio.

O procedimento cirúrgico iniciou com uma incisão na pele crânio lateral na região da articulação fêmuro-tíbio-patelar do membro pélvico esquerdo. Após a incisão de pele realizou-se divulsão do tecido subcutâneo. A artrotomia foi realizada lateralmente permitindo a avaliação articular. A cápsula articular apresentava-se espessada $(5 \mathrm{~mm})$ (Fig.2.A), o sulco troclear raso e patela deslocada medialmente a borda troclear medial. Parte da cartilagem articular da região epicondilar medial encontrava-se erodida pela constante fricção da patela e parte recoberta pela membrana sinovial (pânus) (Fig.2.B). 
Figura 2: Foto da articulação fêmoro-tíbio-patelar esquerda de um gato com luxação patelar medial grau IV.
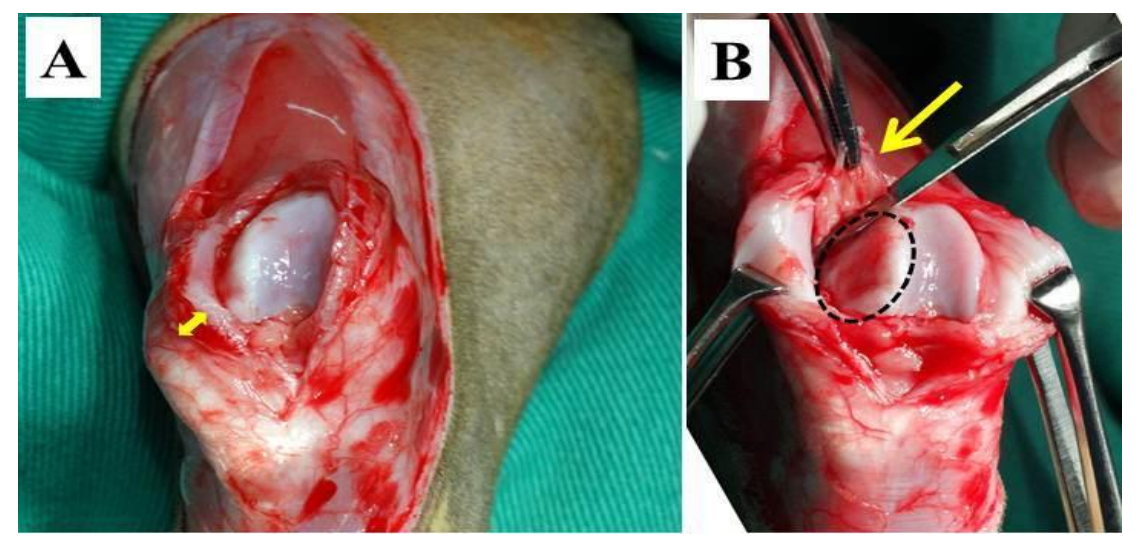

Notar na imagem "A" sulco troclear raso com a patela esquerda deslocada medialmente, tuberosidade tibial desviada medialmente e cápsula articular espessada $(5 \mathrm{~mm})$ (seta amarela). Notas na imagem "B" parte da cartilagem articular da região epicondilar medial erodida (círculo preto) e parte recoberta pela membrana sinovial (seta amarela).

Para tratamento da luxação e realinhamento do mecanismo extensor foram necessárias as técnicas de desmotomia medial, liberação distal parcial do vasto medial e reto femoral (Fig.4.A), trocleopastia (Fig.3), realização de uma sutura anti-rotacional (Fig.5.D), transposição lateral da tuberosidade tibial (Fig.4.B,C,D), imbricação lateral da cápsula articular e reforço do retináculo lateral com fascia Lata (Fig.5). A trocleopastia foi realizada em cunha com o auxílio de um osteótomo, retirando-se de $2 \mathrm{~mm}$ do osso sub-condral (Fig.3).

Figura 3: Foto da articulação fêmoro-tíbio-patelar esquerda de um gato com luxação patelar medial grau IV.

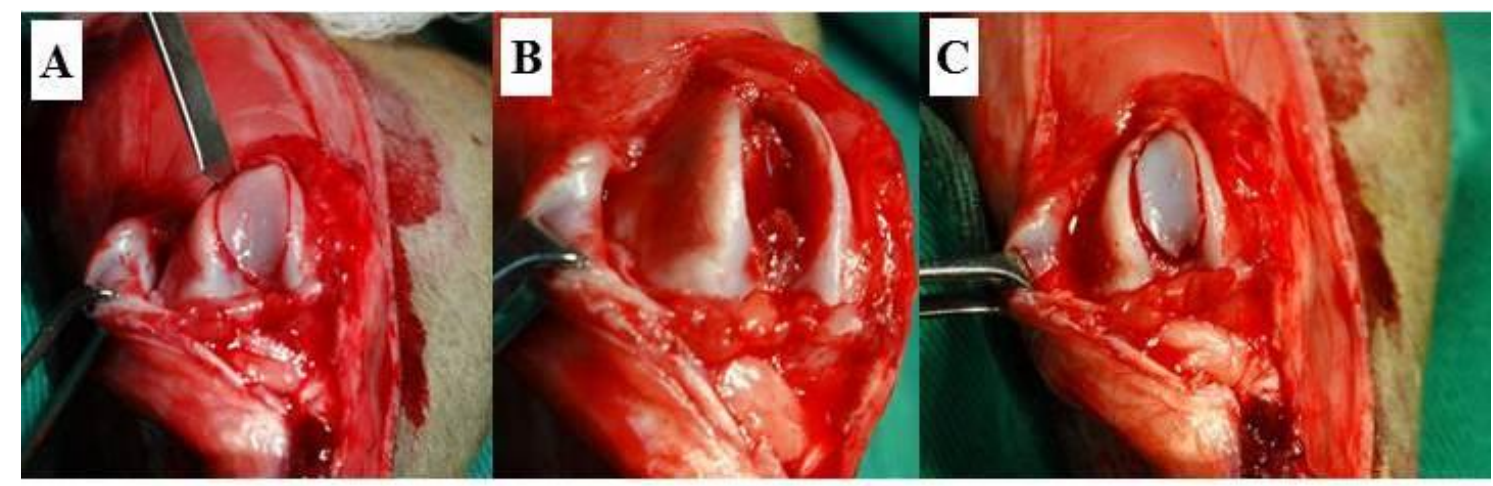

Observar realização da osteotomia em cunha (A), retirada do osso subcondral para aprofundamento do sulco troclear (B) e recolocação da cunha ostocondral (C).

Devido à fibrose e encurtamento do retináculo medial foi necessária sua desmotomia para reposicionamento da patela no sulco troclear. Além disso, foi necessária a liberação parcial distal dos músculos vasto medial e reto femoral uma vez que esses músculos encontravam-se encurtados tracionando a patela medialmente (Fig.4.A). Para correção da rotação interna da tibial foi realizada uma sutura antirotacional ancorada na tuberosidade tibial e na fabela lateral, 
utilizando-se caprofyl 0 (Fig.5.D). Para correção da posição medializada da tuberosidade tibial procedeu-se sua osteotomia e fixação em uma posição lateral a $3 \mathrm{~mm}$, para isso utilizou-se dois fios de kirschner (1mm) (Fig.4.B,C,D).

Figura 4: Foto da articulação fêmoro-tíbio-patelar esquerda de um gato com luxação patelar medial grau IV.

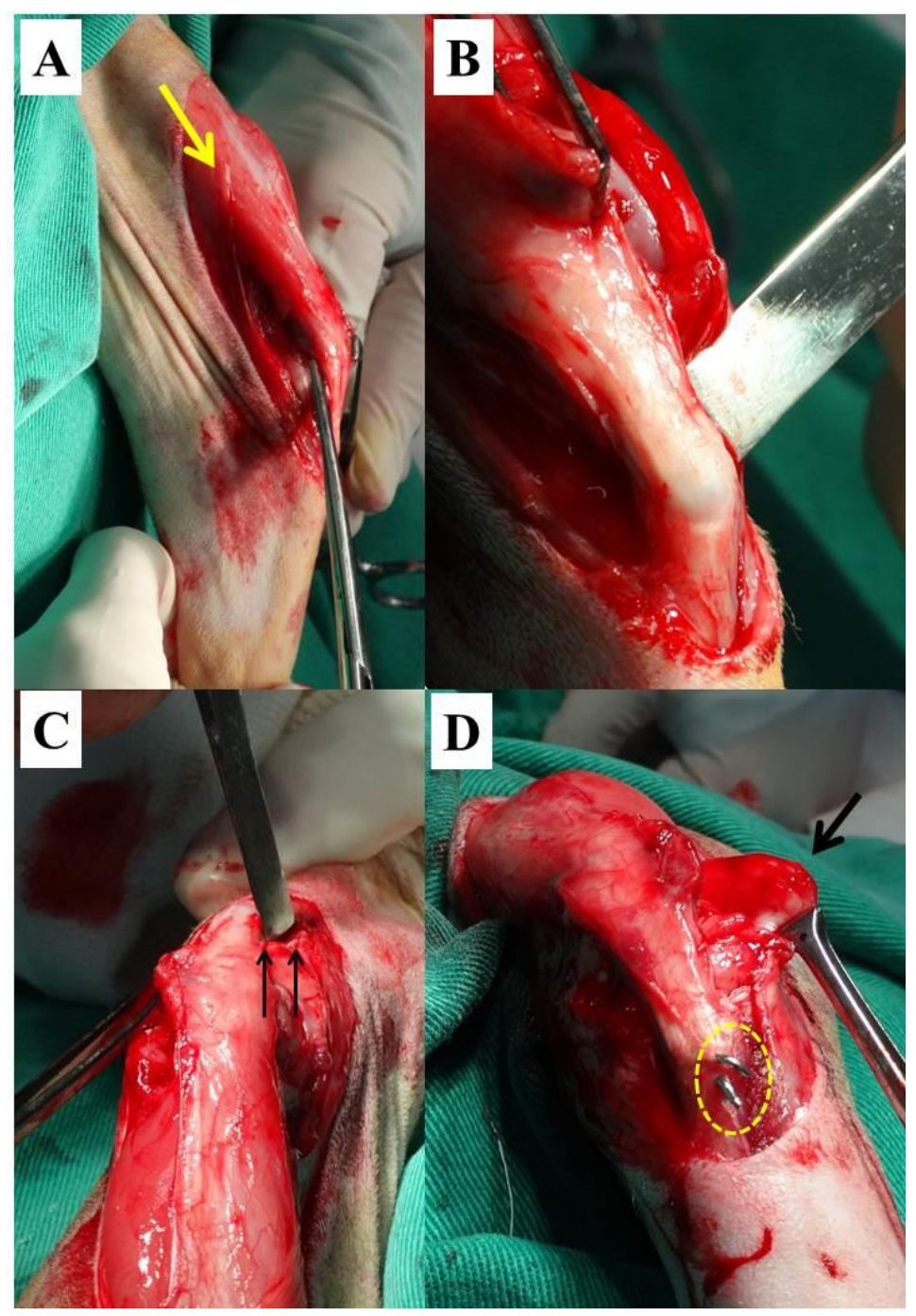

Observar na imagem "A" músculos vasto medial e reto femoral encurtados tracionando a patela medialmente (seta amarela), retináculo medial encurtado (ponta da pinça). Realização da osteotomia da tuberosidade tibial (B), correção de sua posição anormal (B) e sua fixação com 2 fio de Kirschner (C). Notar a frouxidão da porção lateral da cápsula articular (seta preta) (C).

Como a porção lateral da cápsula articular encontrava-se frouxa (Fig.4.D) realizou-se sua imbricação e o reforço do retináculo lateral com retalho de fáscia lata pediculado na patela e ancorado na fabela lateral (Fig.5). 
Figura 5: Foto da articulação fêmoro-tíbio-patelar esquerda de um gato com luxação patelar medial grau IV.

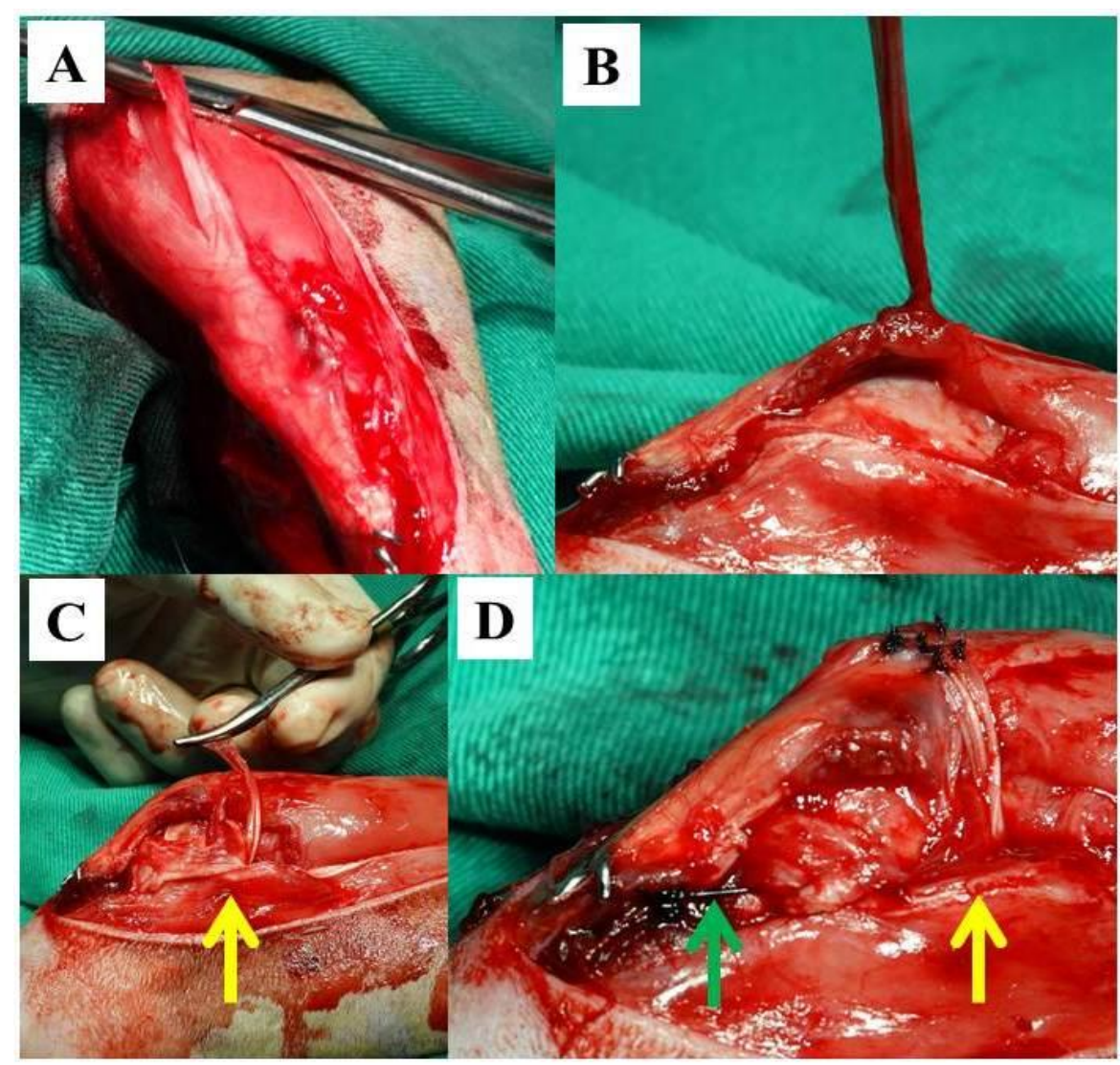

Observar na retirada do retalho de fáscia Lata (A) pediculado na altura do terço médio da patela (B) e ancorado na fabela lateral (C e D). As seta amarelas apontam localização da fabela lateral e a verde parte da sutura anti-rotacional fabelo-tibial.

Todas as suturas internas foram realizadas com caprofyl 3-0, sendo utilizado padrão simples separado na cápsula articular e no reforço lateral do retináculo e simples contínuo na aproximação da fáscia lata e tecido subcutâneo. A dermorrafia foi realizada com nailon 3-0 em padrão simples separado e foi suturada uma gaze sobre a ferida cirúrgica para sua proteção e controle de edema (Fig.6). No pós-operatório o animal recebeu cefalexina (30 mg/kg VO BID) por 7 dias, meloxicam $(0,2 \mathrm{mg} / \mathrm{kg}$ VO SID) por 3 dias e Condroton 1 comprimido VO SID por 180 dias. Também foi realizada uma bandagem de Robert Jones que permaneceu por 10 dias (Fig.6). O animal retornou 48 horas após a cirurgia para verificação da bandagem e em 10 dias para retirada da bandagem e dos pontos de pele. No retorno aos 10 dias o animal apresentava deambulação com claudicação discreta e apoio parcial do membro, a patela estava estável no sulco troclear. No retorno de 20 o animal deambulava normalmente com apoio de peso no membro afetado, havia limitação da flexão total da articulação fêmoro-tíbio-patelar e a patela estava estável no sulco troclear. Aos 60 dias o animal apresentava completa recuperação e recebendo alta médica. 
Figura 6: Fotos do membro pélvico esquerdo de um gato com luxação patelar medial grau IV

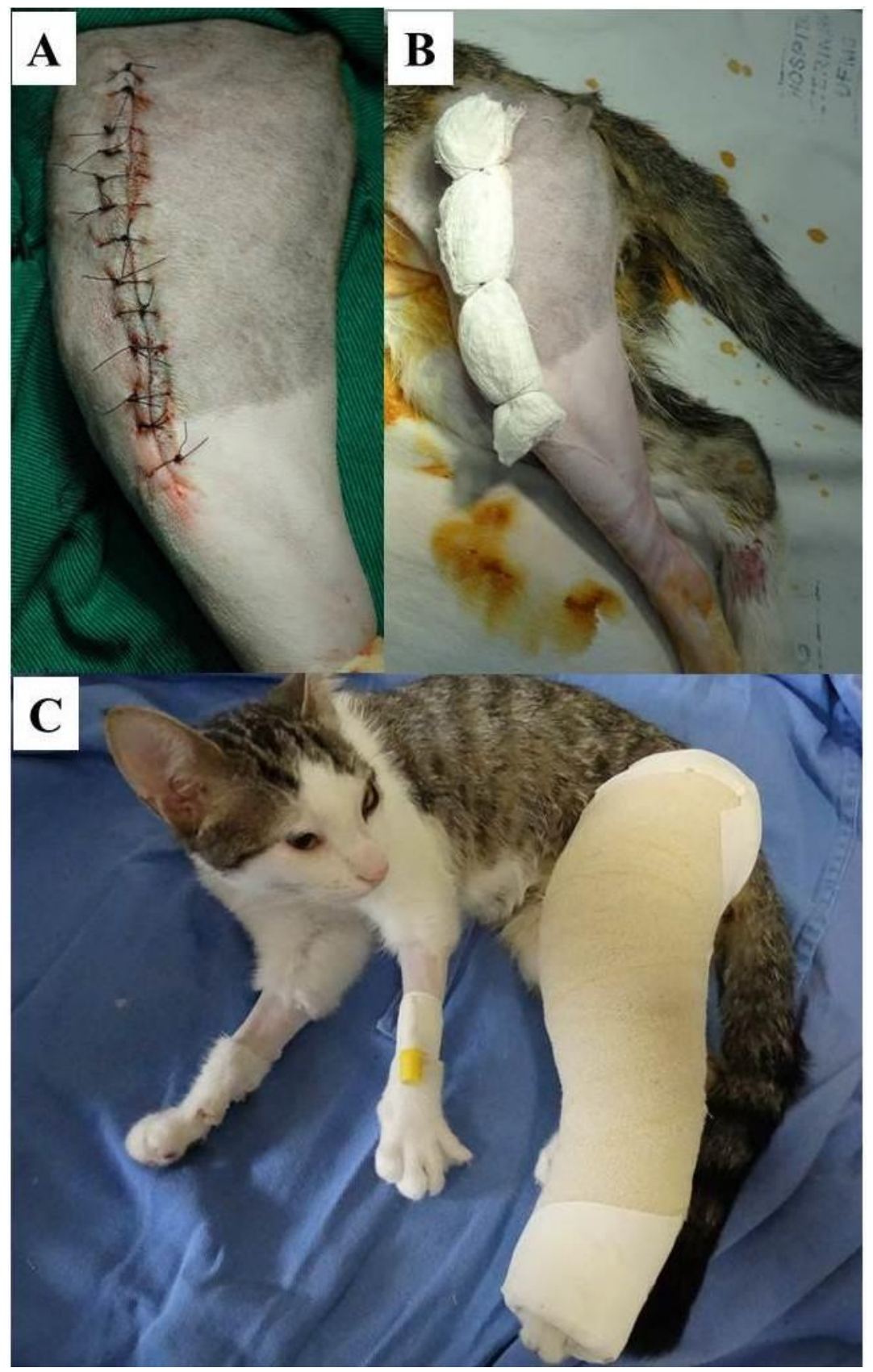

Observar a sutura de pele (A) e a gaze para proteção da ferida cirúrgica (B) e a bandagem de Robert Jones (C).

\section{DISCUSSÃO}

Assim como no presente relato o diagnóstico de luxação patelar deve ser obtido pelo exame clínico observando-se o deslocamento patelar do sulco troclear. Os sinais de luxação patelar medial são variáveis de acordo com as alterações articulares presentes podendo ser observada desde uma claudicação esporádica em animais com grau I até impotência funcional do membro com graves alterações anatômicas no grau IV (SOUZA te al., 2009; SOUZA et al., 2010). Os exames radiográfico ou tomográfico são úteis para a identificação das deformidades a 
serem corrigidas na cirurgia e na detecção da doença articular degenerativa, patologia frequentemente associada as luxações patelares crônicas (TOWLE et al., 2005) e diretamente relacionada ao prognóstico. O deslocamento medial da patelar causa uma distribuição anormal de forças nas placas fisárias de animais em crescimento, podendo causar torsão medial da tíbia proximal, desvio varo do fêmur distal e tíbia proximal (PIERMATTEI et al., 2009). Além disso, o constante atrito da superfície patelar com a cartilagem epicondilar leva a sua erosão e a inflamação crônica que frequentemente desencadeia a doença articular degenerativa, pior complicação das luxações patelares uma vez que é progressiva e não pode ser curada (DOKIC et., 2015). Embora algumas alterações como pânus, espessamento da membrana sinovial e erosão da cartilagem articular estivessem presentes na articulação do paciente aqui relatado, alterações características de doença articular degenerativa ainda não foram observadas. Outra importante patologia associada à luxação medial de patela crónica é a ruptura do ligamento cruzado cranial. A rotação medial da tíbia causa constante estiramento das fibras do ligamento cruzado cranial sobrecarregando-o e predispondo a sua degeneração e ruptura (SCHULZ, 2014). Campbell et al. (2010) observaram ruptura do ligamento cruzado cranial associada a luxação patelar medial (grau IV) em mais de $48 \%$ dos pacientes atendidos com essa patologia no Las Vegas Veterinary Referral Center de 2004 a 2007. No presente relato, a ruptura do ligamento cruzado cranial não ocorreu, provavelmente pelo baixo peso do animal, uso limitado no membro e tratamento em tempo hábil.

A correção da luxação patelar medial é fundamentalmente cirúrgica devendo-se corrigir todas as alterações anatômicas das articulação fêmuro-tibio-patelar e realinhar o mecanismo extensor (SMITH, 2004). Para isso pode ser empregadas as técnicas de reforço lateral do retináculo com fascia lata, imbricação lateral de cápsula articular, desmotomia medial (HOULTON; MEYNINK, 1989), aprofundamento do sulco troclear (WANGDEE et al., 2015), desinserção de grupos musculares (TUDURY et al., 2011), sutura antirrotatória, transposição da tuberosidade tibial (SOUZA et al., 2010), assim como o realizado no presente relato. Mas em casos com desvios angulares importantes em fêmur distal e tíbia proximal e rotação externa da extremidade distal do fêmur são necessárias técnicas de osteotomias corretivas (LARA et al., 2013). Em casos mais graves com intensa doença articular degenerativa e destruição completa da cartilagem articular pode ser tentada a utilização de próteses (DOKIC et., 2015). Embora nenhuma complicação tenha sido observada no presente relato elas podem ocorrer com uma certa frequência. Dentre as possíveis complicações já relatadas estão a reluxação, a migração da cunha osteocondral da trocleoplastia, a migração de implantes, a fratura da tuberosidade tibial, a deiscência da ferida cirúrgica, a inabilidade de extensão completa do membro e a doença articular 
degenerativa (ARTHURS;LANGLEY-HOBBS, 2006; STANKES, STEPHENSON;HAYASHI, 2014).

A bandagem de Robert Jones realizada no pós-operatório tem a função de promover a proteção da ferida cirúrgica, reduzir a amplitude de movimento articular e o edema, dando maior conforto para o paciente, permitindo a cicatrização dos tecidos com menor possibilidade de complicações (PIERMATTEI et al., 2009).

\section{CONCLUSÃO}

Conclui-se que a associação de técnicas cirúrgicas empregadas (reforço lateral do retináculo com fascia lata, imbricação lateral de cápsula articular, desmotomia medial, aprofundamento do sulco troclear, desinserção dos músculos vasto medial e reto femoral, sutura antirrotatória e transposição da tuberosidade tibial) promovem o alinhamento do mecanismo extenso e mantem a estabilidade da patela no suco troclear, sendo efetivas para o tratamento da luxação patelar grau IV em gatos. Cabe ressaltar a importância do tratamento precoce da luxação patelar grau IV, pois forças anormais atuar na placa fisária do animal em fase de crescimento podendo causar graves desvios angulares e torções ósseas que requem técnicas de osteotomias corretivas não empregadas no presente relato.

\section{REFERÊNCIAS}

ARTHURS, G.; LANGLEY-HOBBS, S.J. Complications associated with corrective surgery for patellar luxation in 109 dogs. Veterinary Surgery. v.35, p.559-566, 2006.

CAMPBELL, C.A.et al. Severity of patelar luxation and frequency of concomitant cranial cruciate ligament rupture in dogs: 162 cases (2004-2007). Journal of the American Veterinary Medical Association. v.236, p.887-891, 2010.

DOKIC, Z.et al. Patellar groove replacement in patellar luxation with severe femoro-patellar osteoarthritis. Veterinary and Comparative Orthopaedics and Traumatology. v.28, p.124130,2015

LARA, J.S.et al. Aspectos clínicos, cirúrgicos e epidemiológicos da luxação de patela em cães atendidos no Hospital Veterinário, no período de janeiro de 2000 a julho de 2010: estudo retrospectivo. Arquivo Brasileiro de Medicina Veterinária e Zootecnia. v.65, p.1274-1274, 2013.

HOULTON, J.E.F.; MEYNINK, S.E. Medial patellar luxation in the cat. Journal of Small Animal Practice. v.30, p.349-352, 1989. 
PIERMATTEI, D. L.; FLO, G. L.; DECAMP, C.E. A articulação do Joelho In: Brinker, Piermattei, Flo Ortopedia e tratamento de fraturas de pequenos animais. 4.ed.

Barueri:Manole, 2009. p.637-717

SOUZA, M.M.D.et al. Luxação de patela em cães: estudo retrospectivo. Arquivo Brasileiro de Medicina Veterinária e Zootecnia. v.61, p.523-526, 2009.

SOUZA, M.M.D.et al. Estudo retrospectivo de cães com luxação patelar medial tratados cirurgicamente. Ciência Rural. v.40, n.6, p.1341-1346, 2010.

SCHULZ, K.S. Cirurgias do sistema digestório. In: FOSSUM, T.W. Cirurgia de pequenos animais. $4^{\circ} \mathrm{ed}$. Rio de Janeiro: Mosby Elsevier, 2014. p. 1215-1374.

SMITH, C.W. Luxações de patela. In: HARARI, J. Segredos em cirurgia de pequenos animais. Porto Alegre: Artmed, 2004. p.344-347.

STANKE, N.J.; STEPHENSON, N.; HAYASHI, K. Retrospective risk factor assessment for complication following tibial tuberosity transposition in 137 canine stifles with medial patellar luxation. Canadian Veterinary Journal. v.55, p.349-356, 2014.

TOWLE, H.A.et al. Pré and postoperative radiographic and computed tomographic evaluation of dogs with medial patellar luxation. Veterinary Surgery. v.34, p.265-272, 2005.

TUDURY, E.A.et al. Desinserção proximal do músculo sartório na correção da luxação patelar medial graus III e IV. Arquivo Brasileiro de Medicina Veterinária e Zootecnia. v.63, p.254$527,2011$.

WANGDEE, C.et al. Extended proximal trochleoplasty for the correction of bidirectional patellar luxation in seven Pomeranian dogs. Journal of Small Animal Practice. v.56, p.130-133, 2015. 